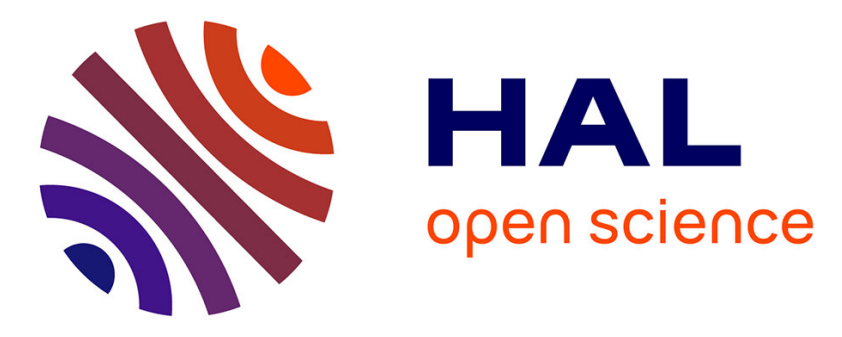

\title{
Proposed behavior for robust header compression over a radio link
}

\author{
Ana Carolina Minaburo Villar, Kamal Deep Singh, Laurent Toutain, Loutfi \\ Nuaymi
}

\section{- To cite this version:}

Ana Carolina Minaburo Villar, Kamal Deep Singh, Laurent Toutain, Loutfi Nuaymi. Proposed behavior for robust header compression over a radio link. ICC 2004: IEEE international conference on communications, June 20-24, Paris, France, Jun 2004, Paris, France. 10.1109/ICC.2004.1313344 . hal-02127728

\section{HAL Id: hal-02127728 \\ https://hal.science/hal-02127728}

Submitted on 24 May 2019

HAL is a multi-disciplinary open access archive for the deposit and dissemination of scientific research documents, whether they are published or not. The documents may come from teaching and research institutions in France or abroad, or from public or private research centers.
L'archive ouverte pluridisciplinaire HAL, est destinée au dépôt et à la diffusion de documents scientifiques de niveau recherche, publiés ou non, émanant des établissements d'enseignement et de recherche français ou étrangers, des laboratoires publics ou privés. 


\title{
Proposed Behavior for Robust Header Compression over a radio link
}

\author{
Ana Carolina Minaburo, Kamal Deep Singh, Laurent Toutain, Loutfi Nuaymi \\ ENST-Bretagne, 2 rue de la châtaigneraie, BP.78, 35510 Cesson-Sévigné, France \\ \{Anacarolina.Minaburo, kd.singh, Laurent.Toutain, Loutfi.Nuaymi \}@enst-bretagne.fr \\ Tel: +33 2.99.12.70.20 Fax: +33 2.99.12.70.30
}

\begin{abstract}
The use of IPv6 in the third generation of cellular networks leads to weak performance of the network systems because the IPv6 header size sent in each packet represents more than twice the payload normally used in video or audio applications. The IETF ROHC (Robust Header Compression) working group has defined a new header compression mechanism to reduce the transmission time and increment the use of the bandwidth in networks with scarce resources. The present version of ROHC considers a static configuration of the ROHC implementation parameters and at the same time does not negotiate some of the link parameters. This paper proposes a dynamic configuration for UMTS radio networks through negotiation. Dynamic configuration enables ROHC to improve its efficiency and robustness based on radio link parameters.
\end{abstract}

\section{INTRODUCTION}

UMTS (Universal Mobile Telecommunication System) Releases 4 and 5 have adopted the use of IPv6 flows for data transmissions. This new version of IP protocol includes many improvements like the number of global addresses and their possible uses in new domains. One problem has yet to be solved: the links and the transmission of IPv6 packets in the radio link are not efficient because of the reduced bandwidth. For example, the real-time applications in cellular networks will use RTP/UDP encapsulation. The header size in an IPv6/UDP/RTP packet is between 60 to 120 bytes while the payload size is 20 bytes or less, considering voice compression algorithms and real time constraints. Header compression algorithms eliminate (or reduce significantly) the redundancy in the header-transmitted information, reducing the header size to much smaller sizes, up to 2 bytes. In UMTS reference protocol architecture, shown in figure 1, a special layer dedicated to header compression has been introduced. This layer is PDCP (Packet Data Convergence Protocol). The header compression mechanisms and their parameters must be negotiated when the channel is established or when the reconfiguration procedure is done. This work is based on the IETF (Internet Engineering Task Force) standardization results of the ROHC working group and, in particular, RFC 3095 [1] and RFC 3241[2]. We have studied the behavior of header compression for real-time applications in UMTS architecture, using UMTS Release $4[3,4]$. In our platform, we have found misbehavior with the PPP (Point-to-Point Protocol) negotiation of $\mathrm{ROHC}$ when used over a radio link. We thus propose a negotiation scheme that enables ROHC to tune its efficiency and robustness in a better way. This paper starts by introducing the ROHC protocol proposed for the header compression of real-time IP applications packets in UMTS. A description of the ROHC negotiation, as suggested in the IETF standard, is given. Then, we describe our proposition for the negotiation of ROHC over UMTS. Finally, some results are given and conclusions for both negotiations are highlighted.

\section{HEADER COMPRESSION IN THE UMTS}

The network functions of the UMTS architecture see figure 1, are divided logically into two service stratums: the AS (Access Stratum) and the NAS (Non Access Stratum). The data flows are divided into two planes: the control and the user plane [5]. The control plane uses the RRC (Radio Resource Control) protocol for the signaling between the network and the UE (User Equipment). The signaling is divided in two in the AS for call control and the NAS for call management.

The PDCP layer exists only in the user plane and it is only used for packet mode services between the UE (User equipment) and the UTRAN (UMTS Terrestrial Radio Access Network). The PDCP layer includes in its functionalities the IP header compression protocols (CTCP [6] and ROHC [1]). The PDCP entities can use zero, one or several header compression mechanisms. In addition, different radio bearers can use the same PDCP entity. The UMTS network has include the ROHC standard as it is used in a PPP network, the negotiation is made initially where the compression parameters are established, but the UMTS network uses its resources dynamically. The RRC layer contains an update procedure to know the QoS parameters values of the radio link at any instant, with which it controls the radio resources. The QoS parameters that are useful for the header compression are the transfer delay, the residual error and the bit error rate, all defined in [7].

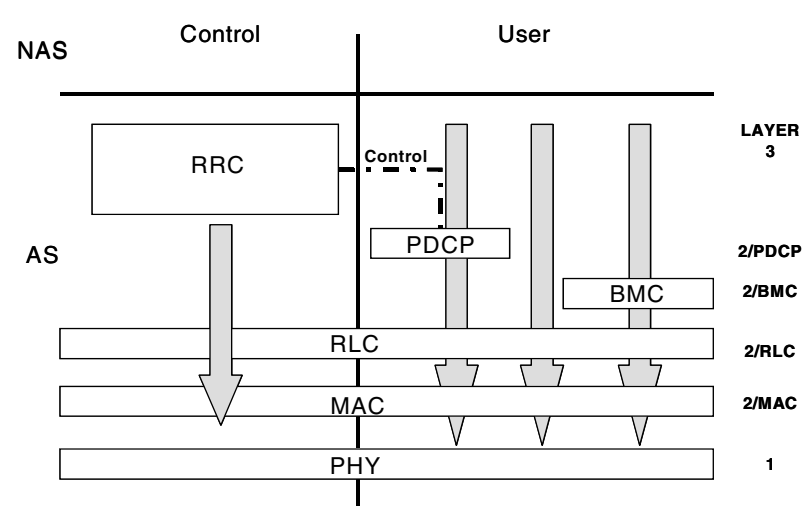

Figure 1. UMTS Radio Interface Protocol Architecture. The PDCP sub-layer is considered only in the user plane. 


\section{ROHC}

The main reason why header compression should be done is the fact that there is redundancy in the header information and between header fields of the same data stream. ROHC mechanism works by removing the redundancy and transfers only changing fields. It classifies the header fields into static and dynamic fields. Static fields are those that remain constant during the lifetime of the packet and dynamic fields are those that keep on changing but their change pattern may be known. The first phase of ROHC protocol is a negotiation. In this phase, the compressor and the decompressor learn about the different characteristics of the link and the parameters that they will use for compression. Negotiation is made while establishing a channel. A channel is a connection between two nodes. Each channel has a compressor and a decompressor at each side, with the possibility of it being either bi-directional or unidirectional. The present solution [2] is for an Internet network where each IP interface in the IP layer can have multiple channels, each one being bi-directional or unidirectional. The present $\mathrm{ROHC}$ negotiation establishes the following parameters:

- MAX_CID: the maximum CID that can be used.

- MAX_Header: the largest header that can be compressed.

- MRRU (Maximum Received Reconstructed Unit): when segmentation is used, it helps to know the maximal size of the segment in bytes.

- Sub-options: there can be zero or several sub-options. Until now, only one sub-option has been specified: The Profile. It informs about the profiles that are supported.

After Negotiation is finished, the ROHC compressor and decompressor start working. The $\mathrm{ROHC}$ compressor removes the redundant header fields and the redundant information in the packet flow. ROHC compression communicates changing fields most of the time. While sending the fields that change, it further achieves efficiency by using an encoding algorithm in which only the last significant bits are sent. The ROHC compressor has three compression levels: Initialization and Refresh (IR), First Order (FO) and Second Order (SO). In IR compression level it tries to establish the static information and in FO compression level it establishes the change pattern of dynamic fields. In the last compression level, SO, it sends encoded values of Sequence Number (SN) and Timestamp (TS) forming the minimal size packets. With the use of this header format packet all header fields can be generated at the other end of the link using the previously established change pattern. When some updates or errors are there, the compressor goes back to the upper compression levels. It only returns to the SO compression level after it has retransmitted the updated information and establishing again the change pattern in the decompressor. The decompressor works at the receiving end of the link and decompresses the headers based on the header fields' information of the context. Both the compressor and the decompressor use a context to store all the information about the header fields. To ensure correct decompression, the context should be synchronized all the time.
The decompressor has three states, the first is No Context (NC) that is when there is no context synchronization, and the second is Static Context (SC) that is reached only after the dynamic information in the context is lost. The third is Full Context (FC), reached when the decompressor has all the information about header fields. When in FC state, the decompressor moves to the initial states as soon as it detects context damage. It uses the $\mathrm{k}$ out of $\mathrm{n}$ rule by looking at the last $\mathrm{n}$ packets, if CRC failures have occurred for at least $\mathrm{k}$ packets then, it assumes context damage and transits backward to an initial state. The decompressor also sends feedback according to the operation modes. ROHC has three operation modes: Unidirectional (U), bi-directional Optimistic (O) and bidirectional Reliable $(\mathrm{R})$. The U-mode is used when the link is unidirectional or when feedback is not possible. For bidirectional links we can use the O-mode or the R-mode. The Omode sends only negative feedbacks, optionally it can also send positive feedbacks but the R-mode uses both negative and positive feedbacks. The decompressor manages the operation mode in which the system will work through the use of mode transitions that allow it to change from one mode to another, based on the link characteristics and the performance requirements. The decompressor also uses some efficient schemes to correct the context when it gets damaged or the synchronization gets lost. The compressor also employs some schemes through which it ensures the correct transmission of the information to the decompressor. These schemes involve many parameters and they control the performance of $\mathrm{ROHC}$ in terms of compression efficiency and robustness.

\section{ROHC COMPRESSION PARAMETERS AND SCHEMES}

The value of the compression parameters of ROHC that determine the efficiency and robustness are not defined in ROHC specification and are not negotiated initially but are stated as implementation dependent. The values of these parameters stay fixed all along the compression. The compression parameters and the schemes that use them, are as follows:

- $\quad L$ : In U-mode and O-mode the ROHC compressor uses a confidence variable $(L)$ in order to ensure the correct transmission of header information.

- $\quad$ Timer_1 (IR_TIMEOUT): In U-mode, the compressor uses this timer to return to the IR compression level and periodically resends static information.

- $\quad$ Timer_2 (FO_TIMEOUT): The compressor also uses another timer in U-mode and this timer is used to go downward to FO compression level if the compressor is working in SO compression level.

- $\quad$ Sliding Window Width $(S W W)$ : The compressor while compressing header fields like Sequence Number (SN) and Timestamp use $W L S B$ encoding that uses a Sliding Window of width equal to $S W W$.

- $\quad W_{-} L S B$ encoding is used to compress those header fields whose change pattern is known. When using this encoding, the compressor sends only the least significant bits. The decompressor uses these bits to construct the original value of the encoding fields. 
- $\quad k$ and $n$ : The ROHC decompressor uses a " $k$ out of $n$ " failure rule, where $k$ is the number of packets received with an error in the last $n$ transmitted packets. This rule is used in the state machine of the decompressor to assume the damage of context and move downwards to a state after sending a negative acknowledgment to the compressor, if bi-directional link is used. The decompressor does not assume context damage and stays in the current state until $k$ packets arrive with error in the last n packets. The $k_{l}$, $n_{l}$ values are used to assume dynamic context damage and $k_{2}, n_{2}$ to assume static context damage.

\section{PROPOSED BEHAVIOR FOR ROHC}

The present negotiation [2] as explained in the third section of this paper does not take into account the characteristics of the radio link layer and the possible constraint in the decompressor. When working in a serial link, problems are avoided because the transmissions are not disrupted by the different possible errors. Important factors in the radio link are the transmission delay, the residual errors and the bit-error-rate (BER) [7]. These parameters can change and the variation change can be high. Negotiation in ROHC must be based on the different characteristics given by the channel state. The compressor needs to know the updated value of these channel attributes to reevaluate the original channel state negotiated initially. Especially when U and O-mode are used because these modes use the $\mathrm{L}$ parameter to increase the robustness of compression.

We propose a negotiation procedure that involves an information exchange between the radio resource control (RRC) layer of UMTS and ROHC (compressor and decompressor). The negotiation begins by requesting the radio parameters from the radio link to the RRC layer through the control link between the PDCP and the RRC, see figure 1. When the compressor has these parameters, it sends the negotiation packet to the decompressor, that will, in its turn, send its parameters through an acknowledge packet.

An update function will be created when using ROHC; this function will receive the values of the QoS parameters of the radio link from the RRC layer. Our negotiation is necessary because in the radio network the terminals are able to know the parameters of only the received radio link, it is thus obligatory for each part to send its radio parameters of its receiving link to the other part, see figure 2 .

In the negotiation packet, the following information will be sent from the compressor to the decompressor:

- MAX_CID: the maximum CID that can be used by compressor;

- MAX_Header: the largest header that can be compressed;

- MRRU (Maximum Received Reconstructed Unit);

- ROHC Profiles supported by the compressor;

- $\quad B E R$ in the compressor side. The BER value is coded to 4 bits according to the UMTS specification [7].
- Transfer delay in the compressor side. This value is coded to 6 bits mapped by the different time limits of the delay in the radio link according to [7].
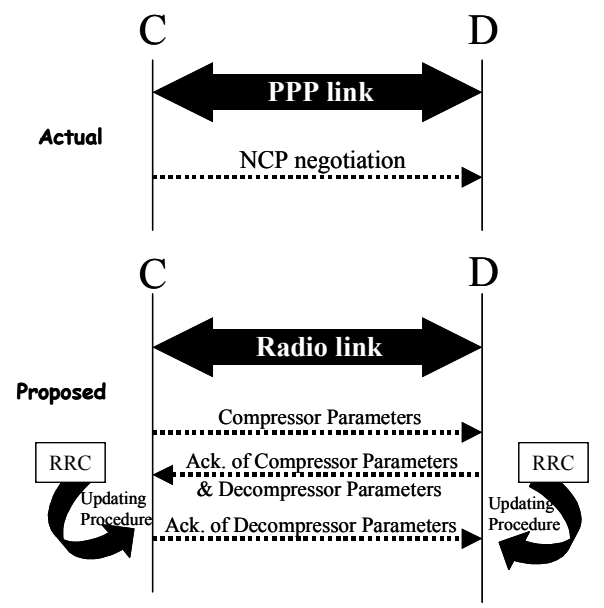

Figure 2. Actual and Proposed ROHC Negotiations. The proposed negotiation gives the opportunity to use the accepted parameters for both sides and also to receive information from the RRC to know the updated link variables.

The decompressor will answer with an acknowledgment packet, reporting the following information:

- MAX_CID (Same as that sent by the compressor if acceptable, otherwise equals to the acceptable value at decompressor side if it is less than that sent by the compressor).

- MAX_Header (Same as that sent by the compressor if acceptable, otherwise equals to the acceptable value at decompressor side if it is less than that sent by the compressor).

- MRRU (Same as that sent by the compressor if acceptable, otherwise equals to the acceptable value at decompressor side if it is less than that sent by the compressor)

- ROHC Profiles supported by the decompressor;

- $\quad B E R$ in the decompressor side;

- Transfer delay in the decompressor side;

If some of the values are unacceptable by the decompressor, it can communicate its possibilities by the acknowledge packet. In the case the decompressor is not able to work in any profile of the compressor, Profile 0 (Non-Compression) is used.

After the negotiation, a dynamic updating procedure is triggered in the RRC to inform the PDCP layer of the new values of the BER and transfer delay through the control link between the PDCP layer and the RRC (see figure 1). When the values of the radio channel attributes change, the compression and decompression variables must be updated (L, timers, SWW, $k, n$ ). We will show in the following section that these compression and decompressor parameter depend on the link characteristics. Using our mechanism, the compressor and the decompressor are sure to know the updated QoS link parameters. This update is frequently (order of magnitude 
$<1$ second) made in the RRC, which will inform the compressor and decompressor if the values have changed.

Knowing the radio link parameters will help to determine the value of L, SWW and timers in the compressor, and the values of $\mathrm{k}$ and $\mathrm{n}$ in the decompressor. If needed, the initial values will be modified in order to improve the performance of ROHC by tuning its efficiency and robustness.

\section{RESULTS}

In order to test the present and the proposed schemes for negotiation (see figure 2), we have developed a UMTS error simulator, which generates UMTS error traces. The error traces are first generated as random sequences based on the error levels of the UMTS radio link [4]. These error traces are fed to our IPv6 ROHC Profile 1 implementation.

Our implementation is the development of all profiles of ROHC header compression. We use Profile 1 to evaluate the performance of ROHC. In our implementation we store the number of packets sent, the average throughput, the number of CRC failed packets in the ROHC implementation and the number of lost packets in the application, the sequence number of every packet sent, the header size in each packet sent and received and the number of each $\mathrm{ROHC}$ packet sent. Our experimental system consists of a video application platform in IPv6 and a PPPoev6 based on FreeBSD4.5 with Kame. The platform is composed of a video application located in both nodes. Through the PPPoe, the client receives the video header compressed packets that will be decompressed by the ROHC decompressor in the other node. At the beginning, node $\mathrm{A}$ is in U-mode. No feedback is sent by node $B$ until the acknowledgment sent by $\mathrm{B}$ changes the operation mode of compressor.

The ROHC negotiation is made when the channel is open between $\mathrm{A}$ and $\mathrm{B}$, where the NCP v6 packet is sent to node $\mathrm{B}$. Then, the ROHC compressor and decompressor starts sending the packets.

\section{A. Dependence of ROHC parameters on link characteristics}

In this paper, results are shown for different values for $\mathrm{L}$ and BER in the O-mode and U-mode see figure 3. The dependence of other ROHC implementation parameters in detail can be seen in [7]. The objective is to test whether the transmission error in the link is reduced when value $\mathrm{L}$ is increased, while taking into account the throughput efficiency because throughput reduces when $\mathrm{L}$ is increased. We use ACL (Average compressed header length) as our benchmark for throughput efficiency so that if ACL is high then throughput is low and vice versa.

We can see the best values of $L$ in the figure 3 and figure 4 (ACL): for a low BER compressor can use a low value of $\mathrm{L}$ and a high value of timers in order to work at maximum throughput without compromising robustness. Thus, for low BER $L=3$ could be used in both U-mode and O-mode. When BER is high, the compressor has to work with high robustness otherwise especially in $U$ mode a long loss event can occur. Thus, $\mathrm{L} \geq 5$ gives the best robustness in $\mathrm{U}$-mode and $\mathrm{L}=5$ in $\mathrm{O}$ - mode. Their differences are shown in figure 3. A compromise in choosing the value of $\mathrm{L}$ has to be made because large value of $\mathrm{L}$ will always decrease the throughput and may not always increase robustness.

In O-mode, decompressor can send a negative acknowledge when error is detected. These negative feedbacks will make an automatic transition to the lower compression level in the compressor.

When incrementing the value of $\mathrm{L}$, the throughput in the link is decremented because each time the compressor goes downwards to FO or to IR compression levels the biggest header format packet are sent L times. On the other hand, it will try to recover the flow if there is an error.
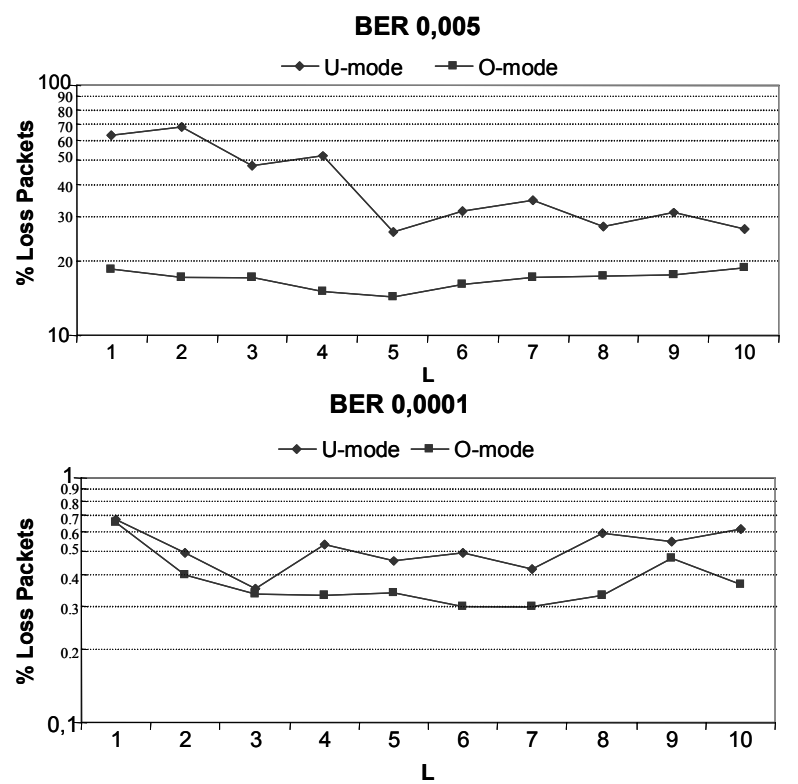

Figure 3. Number of packets lost in the application and in the compression mechanism with different values of $\mathrm{L}$ and BER for O-mode and U-mode; MTU (Maximum Transmission Unit)=1492 bytes. Note that other implementation parameters are already tuned according to [7].

It is important to see that the losses do not change in the same manner as the value of $\mathrm{L}$. The size of the header plays an important role for error probability and the recovering schemes of ROHC when the context is damaged. Figure 3 shows that when $\mathrm{L}$ takes a large value, the transmission losses also increase. The headers in the packets are larger and the errors arrive in consecutive headers, then the recovering schemes fail. In this case, the packets are considered as a CRC fail of ROHC.

\section{B. Optimisation of ROHC performance through negotiation}

The number of packets lost for different values of $\mathrm{L}$ and BER for U-mode is different because the compressor needs to be confident that the decompressor has received the header information. If the compressor keeps a fixed value of $\mathrm{L}$, for example $\mathrm{L}=3$, and since in a radio link the error is (often quickly) variable, a different link error may have a minimal communication error for a different value of $\mathrm{L}$. 
If the compressor takes into account the value of the error in the link and changes accordingly the value of L, the global communication loss could be reduced (figure 4). That is because for U-mode, if an error arrives in the transmission of IR packets a total loss can happen. A total loss is the situation where recovery schemes fail because the context is not established. If the CRC fails, the decompressor will drop all the packets until a time-out to IR state takes place.

Figure 4 shows the comparison our experimentation when the value of $\mathrm{L}$ is fixed to a number all along the flow and when it is variable depending on error, the percentage of loss packets decrement when $\mathrm{L}$ is variable because compressor can update the possible lost information in the decompressor context.

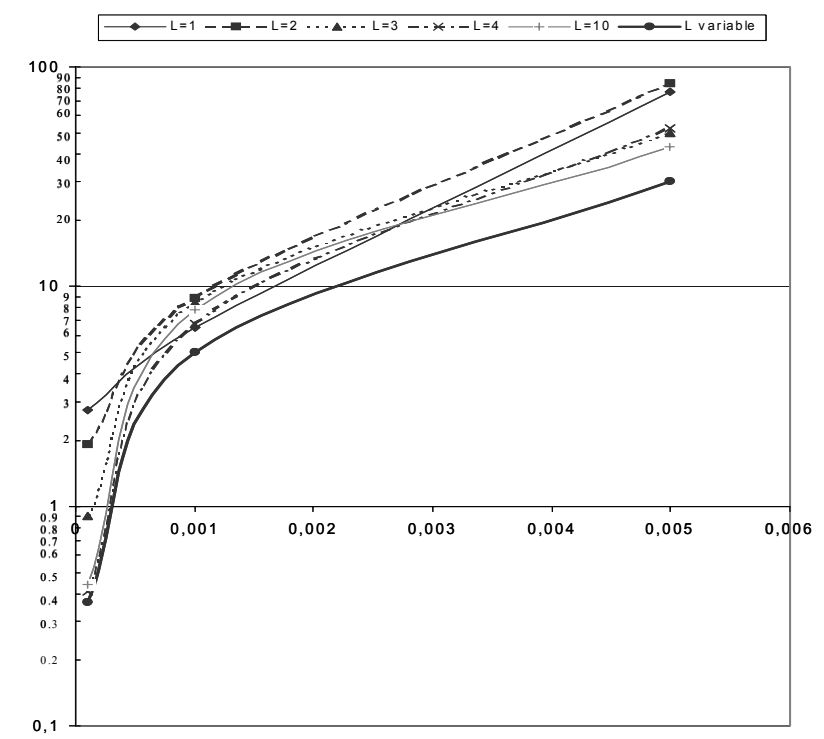

Figure 4. Shows the difference between the actual static negotiation with the dynamic one proposed in this paper for U-mode. The error decreases with a dynamique L, because the number of bytes sent is related to the value of error.

Figure 5 shows the average header length (ACL) of the header send in U-mode and O-mode with different value of error in the link. In U-mode the ACL is proportional direct to $\mathrm{L}$, when $\mathrm{L}=10$ we send 10 times the IR and FO compression levels packets to reinitialize the context, error is not affecting the ACL because compressor always sends the same number of IR and FO level packets if the value of $L$ and timers is fixed.

In O-mode we can see that error increments the ACL value, because each time compressor receives a negative acknowledge IR or FO header packets are send L times. When the error in the link is low the ACL remains in SO compression level. In the other hand when error is high, the ACL of O-mode presents the best result only with some values of $\mathrm{L}(\mathrm{L}=5$ or 6$)$, in the other cases ACL increases: When $L<5$ because context is lost and compressor needs to send IR or FO packets more frequently and when $\mathrm{L}>6$ because we send $\mathrm{L}$ times IR and FO packets each time a negative feedback is sent. We have also measured the ACL when L remains variable; we have noticed that it becomes optimal because the compressor minimized the number of IR and FO compression level packets sent.

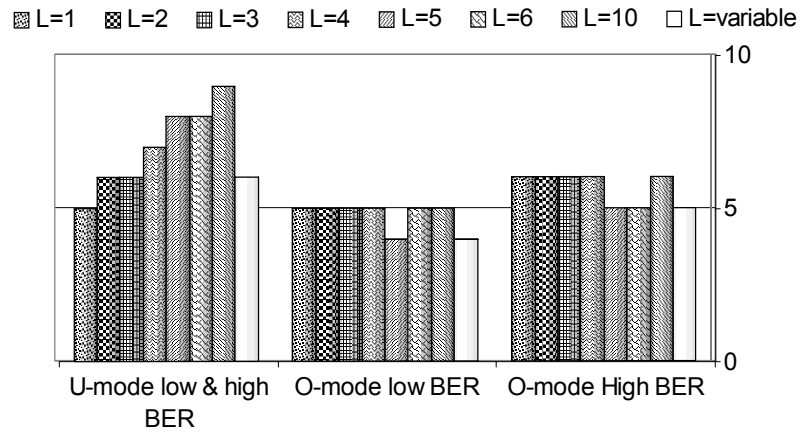

Figure 5. The ACL of the Static vs Dynamique configuration for different values of $\mathrm{L}$ and BER. Note that 2 bytes are extra because UDP checksum is mandatory for IPv6.

\section{CONCLUSION}

In this paper, we have seen the importance of link parameters negotiation for the performance of ROHC over a radio link. We notice that the system has generally the same response when the error in the link is relatively small but when this error increases, the response to the different values of the compression parameters is different. The smallest and largest values for the compression parameters give a larger loss due to different causes. It is always possible to set a range of values where the transmission error is minimized and the throughput maximized.

For the U-mode and the O-mode a compromise has to be made for the choice of L. The curve of figure 3 is a good motivation for using variable compression parameters when the error is large in the link. If a UE keeps a fixed value for the compression parameters, the degradation in the user application leads to a bad performance for the protocol even though it has demonstrated a large robustness against error. We thus recommend the negotiation and update of link parameters like BER and transfer delay in order to improve the performance of ROHC over radio links. Future work will be to analyze the behavior of $\mathrm{ROHC}$ in a real radio stack.

\section{REFERENCES}

[1] Bormann C., et Al., "RObust Header Compression (ROHC): Framework and four profiles: RTP, UDP, ESP and uncompressed", IETF RFC 3095, 2001.

[2] Bormann C., "ROHC over PPP”, IETF RFC 3241, 2002.

[3] 3GPP, Technical Specification Group Radio Access Network, "Packet Data Convergence Protocol (PDCP) Specification", 3GPP TS25.323, 2002.

[4] 3GPP, Technical Specification Group Radio Access Network, "Radio Resource Control (RRC) Protocol Specification”, 3GPP TS25.331, 2002.

[5] Holma H., Toskala A., "WCDMA for UMTS", Wiley, England 2001.

[6] Degermark M., et. Al., "IP Header Compression", IETF RFC 2507, 1999.

[7] 3GPP, Technical Specification Group Core Network, "Mobile Radio Interface Layer 3 Specification”, 3GPP TS24.008, 2002.

[8] Minaburo, A., et. Al., "Configuration and Analysis of Robust Header Compression in UMTS”, IEEE PIMRC'03, Beijing Chine, 2003. 